\title{
Aptitude au séchage des fruits de quelques variétés de manguiers cultivées au Cameroun
}

\author{
Anselme Kameni $^{\mathrm{a} *}$, Carl Moses Mbofung ${ }^{\mathrm{b}}$, Zenabou Ngnamtam ${ }^{\mathrm{b}}$, Jean Doassem ${ }^{\mathrm{a}}$, Layla Hamadou ${ }^{\mathrm{a}}$
}

a Irad, BP 415, Garoua,

Cameroun

${ }^{\mathrm{b}}$ Ensai, BP 455, Ngaoundéré, Cameroun

* Correspondance et tirés à part

Fruits, 2003, vol. 58, p. 89-98 (C) 2003 Cirad/EDP Sciences All rights reserved DOI: 10.1051/fruits:2002039

RESUMEN ESPAÑoL, p. 98

\section{Aptitude for drying of fruit produced by some varieties of mango trees cultivated in Cameroon.}

Abstract - Introduction. In Cameroon, the production of mango trees (Mangifera indica) is significant during the harvest period which lasts (2 to 3) month year ${ }^{-1}$, according to the area. In rural zones, there is therefore a problem of overproduction. Work has shown that the drying of mango would be an interesting solution for the conservation of this overproduction. In rural zones, electric dryers which allow a fast and constant drying cannot be used because of the absence of electricity. To encourage the producers to use drying techniques to conserve the surplus mangos in certain seasons, the aptitude for drying of some varieties of mangos produced in Cameroon was studied by comparing the products obtained by electric or solar drying. Materials and methods. Fruits of three improved varieties of mango (Amélie, Zill and Irwin) and of a local variety (Horé Wandou) were collected with two degrees of maturity: commercial maturity and advanced maturity. After preparation of the fruits, the pulp was cut into slices and was dried either by solar drier, or by direct exposure to the sunshine. The physicochemical composition of the pulp samples was studied before and after drying. Results and discussion. After drying at $50^{\circ} \mathrm{C}$ for $24 \mathrm{~h}$ (electric dryer), the dried mango slices presented a water content ranging between $(16$ and 24$) \%$. Significant differences appeared in the outputs with the drying of the studied varieties. The contents of the various analyzed physicochemical components varied according to the varieties and the degree of maturity of the collected fruits. The average rates of the component (vitamin $\mathrm{C}$, reducing sugars, soluble solids) conservation after drying were higher than $56 \%$, except for the fiber content, whose conservation rate was $16 \%$. The mangos dried directly in the sun were similar to those obtained with an electric dryer, in spite of a longer drying time and a lower amount of vitamin C. Conclusion. The varieties Amélie, Zill, Irwin and the local variety Horé Wandou lend themselves well to drying, which preserves the principal nutritive elements with good outputs.

Cameroon / Mangifera indica (fruits) / varieties / keeping quality / drying / dryers / physicochemical properties

\section{Aptitude au séchage des fruits de quelques variétés de manguiers cultivées au Cameroun.}

Résumé - Introduction. Au Cameroun, la production du manguier (Mangifera indica) est forte pendant la période de récolte qui dure (2 à 3) mois $\mathrm{an}^{-1}$ suivant les régions. En zone rurale, il y a alors un problème de surproduction. Des travaux ont montré que le séchage de la mangue serait une voie intéressante pour la conservation de cette surproduction. En zone rurale, les séchoirs électriques qui permettent pourtant un séchage rapide et constant ne peuvent être utilisés du fait de l'absence d'électricité. Afin d'inciter les producteurs à utiliser le séchage comme technique de conservation des mangues excédentaires en certaines saisons, l'aptitude au séchage de quelques variétés de mangues produites au Cameroun a été étudiée en comparant les produits obtenus par séchage électrique ou solaire. Matériel et méthodes. Les fruits de trois variétés de mangue améliorées (Amélie, Zill et Irwin) et d'une variété locale (Horé Wandou) ont été récoltés à deux degrés de maturité : maturité commerciale et maturité avancée. Après préparation des fruits, la pulpe a été découpée en lamelles et séchée soit par séchoir solaire, soit par exposition directe au soleil. La composition physico-chimique des échantillons de pulpe a été étudiée avant et après séchage. Résultats et discussion. Après un séchage à $50^{\circ} \mathrm{C}$ pendant $24 \mathrm{~h}$ (séchoir électrique), les lamelles de mangue séchée obtenues ont présenté une teneur en eau comprise entre (16 et 24)\%. Des différences significatives sont apparues dans les rendements au séchage des variétés étudiées. Les teneurs des différents constituants physico-chimiques analysés ont varié en fonction des variétés et du degré de maturité des fruits récoltés. Les taux moyens de conservation des constituants (vitamine $\mathrm{C}$, sucres réducteurs, extraits secs solubles) après séchage ont été supérieurs à $56 \%$ sauf pour la teneur en fibres dont le taux de conservation a été de $16 \%$. Les mangues séchées directement au soleil ont été semblables à celles obtenues par passage en séchoir électrique malgré une durée de séchage plus longue et un moindre taux en vitamine C. Conclusion. Les variétés Amélie, Zill, Irwin et la variété locale Horé Wandou se prêtent bien au séchage qui conserve les principaux éléments nutritifs avec des rendements importants.

Cameroun / Mangifera indica (fruits) / variété / aptitude à la conservation / séchage / séchoir / propriété physico-chimique 


\section{Introduction}

Au Cameroun, la production du manguier (Mangifera indica) est forte pendant la période de récolte qui dure de (2 à 3) mois $\cdot a^{-1}$ suivant les régions. Les grandes quantités de mangues alors disponibles sur les marchés entraînent des méventes et de fortes pertes après-récolte [1]. Les problèmes de maladies fongiques, la sensibilité du fruit à une réfrigération prolongée et sa courte durée de vie limitent son transport vers les marchés éloignés de sa zone de production. Il y a donc un réel problème de conservation.

Des technologies de transformation de pulpe en jus et nectars, ainsi que celles de fabrication de gelées et confitures ont été expérimentées [2-4]. Cependant, le stockage des jus en milieu rural se heurte aux problèmes de réfrigération. Dans de telles conditions, des travaux [5-8] ont montré que le séchage de la mangue est une voie intéressante. Le grand nombre de variétés de mangues cultivées - variétés d'Inde monoembryoniques ou polyembryoniques, différences morphologiques et physicochimiques, etc. -, justifie que l'aptitude au séchage des mangues soit mieux étudiée. En zone rurale, les séchoirs électriques qui permettent pourtant un séchage rapide et constant ne peuvent être utilisés du fait de l'absence d'électricité. En revanche, dans les régions chaudes du nord du Cameroun et dans les pays des savanes d'Afrique, le séchage solaire est possible et facilité par des conditions favorables d'ensoleillement, de température et d'humidité relative.

Dès lors, le séchage solaire de la mangue pourrait être une technique intéressante pour réduire les pertes après-récoltes et développer de nouveaux produits pour les marchés urbains. Notre travail a donc cherché à étudier l'aptitude de quatre variétés de mangue en comparant les caractéristiques des produits obtenus soit par séchage électrique, soit par séchage solaire dans les conditions du nord du Cameroun.

\section{Matériel et méthodes}

\subsection{Matériel végétal}

Les mangues utilisées ont été collectées sur un marché local et dans deux vergers de la région de Kismatari : le verger expérimental de la station Irad (Institut agricole pour le développement) de Garoua et un verger privé.

Sur le marché local, les fruits récupérés ont été identifiés comme issus d'une variété locale, la variété "Horé Wandou "; ils se trouvaient être à maturité dite commerciale qui permet de disposer de fruits mûrs et encore bien fermes. La variété Horé Wandou est considérée comme une variété intermédiaire entre précoce et tardive avec une tendance tardive plus accentuée.

En vergers, les fruits analysés, de maturité commerciale (fruits fermes) ou avancée (fruits très tendres), ont été prélevés sur des manguiers connus comme précoces variétés Amélie et Irwin - ou intermédiaires (variété Irwin)

Le choix des mangues échantillonnées a donc été guidé par le type de variété, soit amélioré (Amélie, Irwin et Zill), soit local (Horé Wandou), et par le type de maturité la caractérisant (précoce ou tardive). Selon les variétés, les caractéristiques de forme, poids et couleur des fruits (vert au jaune orangé) ont varié. Elles sont restées proches de celles déjà observées pour des mangues semblables [9].

\subsection{Préparation des échantillons}

Après tri des fruits collectés, les mangues retenues ont été lavées, égouttées puis pesées (figure 1). Le poids des fruits ainsi échantillonnés a été de $(370,76 \pm 67,50) \mathrm{g}$ pour Amélie, $(313,99 \pm 18,70) \mathrm{g}$ pour Zill, $(264,38 \pm 22,50) \mathrm{g}$ pour Irwin et $(154,79 \pm$ $19,20)$ g pour la variété Horé Wandou. La largeur des fruits a été comprise entre (10,1 et 7,47$) \mathrm{cm}$ et la hauteur entre $(7,20$ et $12,60) \mathrm{cm}$.

Les fruits ont alors été pelés, dénoyautés puis découpés en lamelles. La peau, les noyaux et la pulpe ont été pesés séparément. Les lamelles de mangue ont été ensuite séchées soit en séchoir électrique soit par une technique traditionnelle de séchage au soleil.

Le séchoir électrique utilisé a été un séchoir Turbo mixte à air pulsé chaud de 
marque Bonnet, Type TMN. Les tranches de mangue ont été disposées sur des claies recouvertes d'une toile ; elles ont été suffisamment espacées (figure 2) pour permettre la circulation de l'air entre elles ; leur densité sur la toile a été en moyenne de $4 \mathrm{~kg} \cdot \mathrm{m}^{-2}$. Les claies ont été placées en séchoir réglé à $50^{\circ} \mathrm{C}$. La convection de l'air chaud permet à la fois de chauffer le produit et de transporter la vapeur dégagée vers l'extérieur. Les tranches ont été retournées trois à quatre fois pendant le séchage de façon à éviter que les lamelles de mangue n'adhèrent à la toile. Les lamelles ont été jugées sèches après $24 \mathrm{~h}$ de traitement dans un tel séchoir [7] ; à ce stade, elles sont fermes à la pression des doigts et ne sont plus collantes.

Le séchage solaire direct des lamelles de mangue est celui qui peut être utilisé par des petits producteurs isolés. Cette technique a été testée avec les fruits des variétés Amélie et Irwin. Les lamelles de pulpe ont été réparties sur des claies de séchage solaire de $190 \mathrm{~cm}$ de longueur, $90 \mathrm{~cm}$ de largueur et $5 \mathrm{~cm}$ de profondeur. Ces claies sont constituées d'un cadre en bois recouverts d'une toile de type moustiquaire en matière synthétique, sur laquelle les tranches ont été disposées. L'empilement de telles claies a permis de protéger les lamelles de mangues des attaques extérieures (mouches, abeilles, etc.). L'ensemble déposé sur un support d'environ $1 \mathrm{~m}$ de haut a été directement exposé au soleil pendant $27 \mathrm{~h}$ de rayonnement solaire, soit pendant (3 à 4) j en moyenne. À ce stade, le poids des lamelles est resté stable.

Après séchage, les lamelles ont été pesées et les rendements calculés à partir du rapport [poids sec / poids frais].

\subsection{Analyses physico-chimiques des mangues}

Différentes caractéristiques physico-chimiques ont été mesurées dans la pulpe frâ̂che et la pulpe séchée. Pour étudier les teneurs dans la pulpe séchée, $1 \mathrm{~g}$ d'échantillon a été ramolli par trempage dans $10 \mathrm{~mL}$ d'eau distillée pendant (30 à 45) min puis les mesures ont été effectuées comme pour de la pulpe fraîche.
La teneur en eau des échantillons a été déterminée par étuvage à $105^{\circ} \mathrm{C}$ pendant

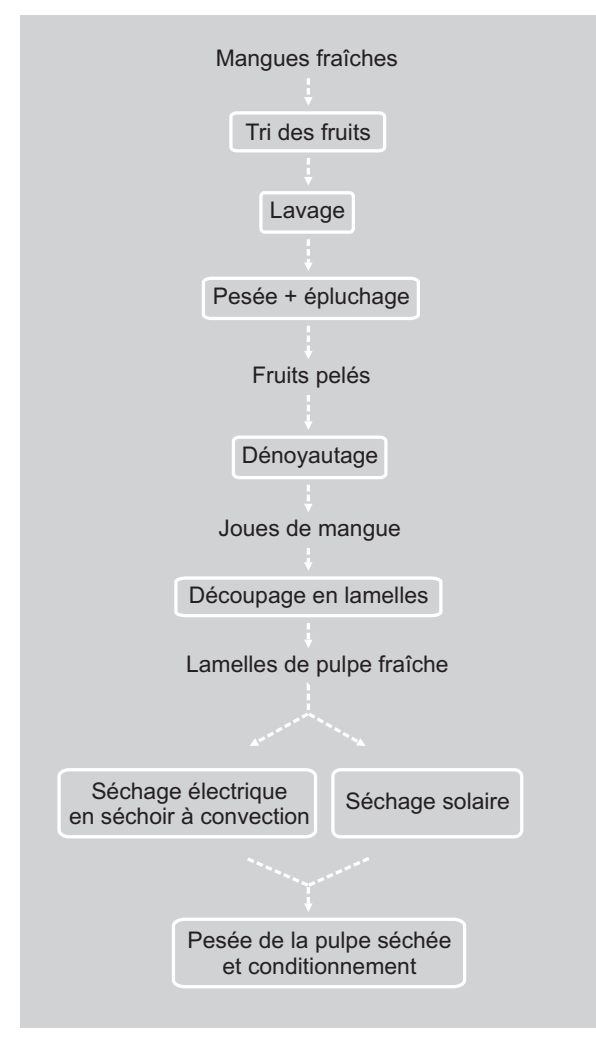

Figure 1.

Schéma de production de lamelles de mangue séchée au nord du Cameroun.
Figure 2.

Étalage de lamelles de mangue fraîche sur une claie avant séchage solaire (Cameroun, photo Irad 2002).

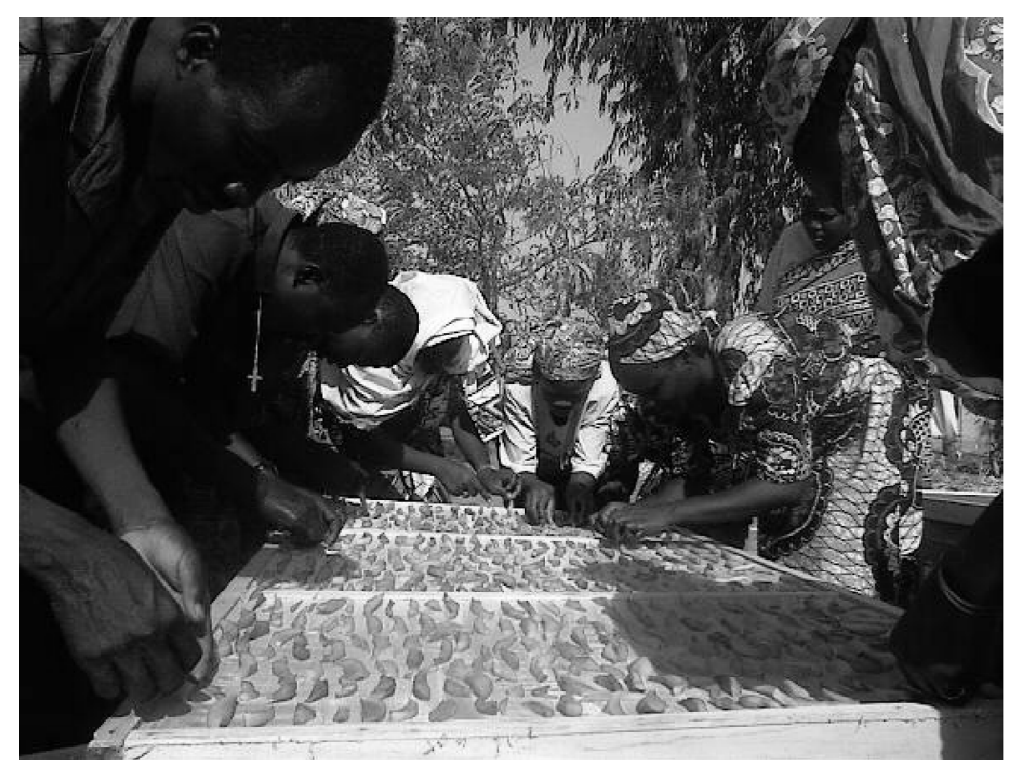


$24 \mathrm{~h}$ [10]. Le pH des échantillons a été mesuré au pH-mètre. L'acidité titrable a été déterminée par la méthode normée [10] avec la phénolphtaléine comme indicateur coloré et la soude $(\mathrm{NaOH} 0,1 \mathrm{~N})$ comme base. La teneur en vitamine $\mathrm{C}$ a été évaluée par la méthode à l'indophénol [11].

La teneur en fibre a été estimée par traitement des échantillons par ébullition acide dans une solution d'acide sulfurique ; le résidu obtenu a été lavé à l'eau puis soumis à une ébullition basique dans une solution d'hydroxyde de sodium avant d'être à nouveau lavé, puis filtré et séché en étuve à $103 \pm 2^{\circ} \mathrm{C}$ pour être enfin incinéré au four à $600^{\circ} \mathrm{C}$ pendant une période variant de 30 min à $1 \mathrm{~h}$.

La détermination du taux de sucres réducteurs a été effectuée par la méthode colorimétrique au DNS (acide 3,5 dinitro salicylique). Les extraits secs solubles des échantillons ont été mesurés au réfractomètre.

Les taux de conservation des différents constituants de la mangue après séchage ont été calculés par rapport à l'unité de mangue fraîche en appliquant la formule

$$
\left[\left(Q_{\text {sèche }} / Q_{\text {fraîche }}\right) \times 100\right] \text {, }
$$

avec $\mathrm{Q}_{\text {sèche}}$, quantité de matière dans la mangue séchée et $Q_{\text {fraîche, quantité de }}$ matière dans la mangue fraîche.

\subsection{Analyses statistiques}

Le dispositif expérimental utilisé a été un schéma factoriel en blocs complètement randomisés. Les facteurs étudiés ont été le degré de maturité (commerciale ou avancée) et la variété considérée (Amélie, Zill, Irwin et la variété locale). Un bloc a été constitué par les mangues d'une même récolte. Au total six séries de récolte ont été effectuées. L'analyse de variance a été effectuée avec le logiciel Statitcf [12].

\section{Résultats}

La composition chimique de la pulpe de mangue fraîche a varié en fonction de la variété et du degré de maturité du fuit analysé (tableau I).
Le $\mathrm{pH}$, qui a oscillé entre 3,7 et 4,2, a augmenté avec le degré de maturité pour les trois variétés améliorées mais non pour la variété locale. L'acidité titrable a varié d'une variété à l'autre et a été moindre dans les fruits à maturité avancée que dans ceux à maturité commerciale. La teneur en eau des fruits a été globalement comprise entre (74 et 81$) \%$. Cependant, quel que soit le degré de maturité des fruits, les variétés Irwin et Amélie ont eu des teneurs en eau supérieures à celles des variétés Zill et locale. La teneur en extraits secs solubles a peu changé en fonction du degré de maturité du fruit mais elle montre des différences significatives d'une variété à l'autre, les variétés Amélie et Zill ayant les taux les plus élevés. Pour une même variété, les teneurs en eau, sucres réducteurs et vitamine $\mathrm{C}$ ont augmenté dans le fruit avec le degré de maturité. À maturité commerciale, les fruits de la variété Zill sont les plus acides alors que ceux de la variété Irwin sont les plus riches en vitamine C. La pulpe de la variété locale a été la plus riche en fibres $(1,73 \%)$ et celle de la variété Amélie la moins fibreuse $(0,70 \%)$.

En séchoir électrique, les rendements de matière sèche (ms) par rapport au poids de fruits frais (mf) des différentes variétés de mangue ont varié de $(10,58$ à $14,82) \mathrm{kg} \mathrm{ms} \cdot 100 \mathrm{~kg}^{-1}$ de $\mathrm{mf}$, tous degrés de maturité confondus (tableau II) ; ils n'ont pas été significativement différents d'une variété à l'autre. En séchage solaire, ces rendements ont été analogues. En revanche, des différences significatives sont apparues, entre variétés, pour les rendements en matière sèche obtenus après séchage de la pulpe. Ainsi, la pulpe des fruits de la variété Zill a eu un rendement relativement plus élevé au séchage que celle des variétés Amélie et Irwin. Cela pourrait être une conséquence de la forte teneur en eau des fruits de ces dernières variétés de composition différente de ceux de Zill en revanche plus riches en sucre. Les taux de conservation de la matière sèche des fruits après un bilan de masse ont varié de $60,25 \%$ pour les mangues Amélie à 81,62\% pour les mangues locales. Des pertes de matière n'ont pas été 
Tableau I.

Composition physico-chimique de la pulpe de mangues fraîches en fonction de la variété considérée et de son degré de maturité (Cameroun).

\begin{tabular}{|c|c|c|c|c|c|c|c|}
\hline Variété de mangue & $\mathrm{pH}$ & $\begin{array}{l}\text { Acidité titrable } \\
\left(\mathrm{mEq} \cdot 100 \mathrm{~g}^{-1}\right)\end{array}$ & $\begin{array}{c}\text { Teneur en eau } \\
\text { (\%) }\end{array}$ & $\begin{array}{l}\text { Extraits secs solubles } \\
\text { ('Brix) }\end{array}$ & $\begin{array}{c}\text { Sucres réducteurs } \\
(\%)\end{array}$ & $\begin{array}{l}\text { Vitamine C } \\
\left(\mathrm{mg} \cdot 100 \mathrm{~g}^{-1}\right)\end{array}$ & $\begin{array}{c}\text { Fibres } \\
(\%)\end{array}$ \\
\hline \multicolumn{8}{|c|}{ Maturité commerciale } \\
\hline Amélie & $3,75 b$ & $12,8 b$ & $79,25 a$ & $12,58 \mathrm{c}$ & $3,82 \mathrm{~b}$ & 37,33 a & $0,70 \mathrm{c}$ \\
\hline Zill & $3,85 b$ & $16,2 \mathrm{a}$ & $74,03 \mathrm{~b}$ & $22,0 \mathrm{a}$ & $4,87 \mathrm{~b}$ & $11,38 d$ & $1,08 \mathrm{~b}$ \\
\hline Irwin & $3,79 b$ & $10,0 \mathrm{c}$ & $80,41 \mathrm{a}$ & $18,75 b$ & $4,61 \mathrm{~b}$ & $17,25 \mathrm{~b}$ & $1,04 \mathrm{~b}$ \\
\hline Locale & $4,03 \mathrm{a}$ & $10,0 \mathrm{c}$ & $77,40 \mathrm{~b}$ & $21,42 \mathrm{a}$ & $6,55 \mathrm{a}$ & $14,35 \mathrm{c}$ & $1,73 \mathrm{a}$ \\
\hline \multicolumn{8}{|l|}{ Maturité avancée } \\
\hline Amélie & $4,11 \mathrm{a}$ & $5,5 \mathrm{e}$ & $80,37 \mathrm{a}$ & $15,83 \mathrm{c}$ & $5,53 \mathrm{c}$ & $48,13 b$ & - \\
\hline Zill & $4,15 a$ & $7,8 \mathrm{~d}$ & $76,89 \mathrm{~b}$ & $21,2 \mathrm{a}$ & $7,48 \mathrm{~b}$ & $14,81 \mathrm{~d}$ & - \\
\hline Irwin & $4,17 \mathrm{a}$ & $5,8 \mathrm{e}$ & $80,89 a$ & $17,17 b$ & $6,61 \mathrm{~b}$ & $59,20 \mathrm{a}$ & - \\
\hline Locale & $3,99 a$ & $8,3 d$ & $75,20 \mathrm{~b}$ & $21,67 \mathrm{a}$ & $9,30 \mathrm{a}$ & $34,29 \mathrm{c}$ & - \\
\hline $\begin{array}{l}\text { Pour une caractéris } \\
(5 \%) .\end{array}$ & ue phy & sico-chimique do & hée, les valeurs & suivies d'une même lettre & ne sont pas signifi & tivement diffé & ntes \\
\hline
\end{tabular}

observées pendant les opérations de séchage. Toutefois, des réactions d'hydrolyse des polyfructanes et de la pectine contribuent à une importante libération d'eau qui s'évapore pendant le séchage et dimi- nuerait les rendements attendus en théorie. La libération d'éthylène et de $\mathrm{CO}_{2}$ pendant la respiration encore active au début du séchage contribuerait aussi à réduire les rendements. Ces changements et échanges

\section{Tableau II.}

Rendements en matière sèche des mangue de différentes variétés après séchage (Cameroun) (kg de matière sèche / $100 \mathrm{~kg}$ de fruits frais).

\begin{tabular}{|c|c|c|c|c|c|}
\hline \multirow[t]{2}{*}{ Variété de mangue } & \multicolumn{2}{|c|}{ Séchoir électrique } & \multicolumn{2}{|c|}{ Séchage solaire } & \multirow[t]{2}{*}{$\%$ de matière sèch } \\
\hline & Fruit & Pulpe & Fruit & Pulpe & \\
\hline Amélie & $10,58 \mathrm{a}$ & $14,65 \mathrm{c}$ & $11,40 \mathrm{a}$ & $16,38 \mathrm{~b}$ & 60,25 \\
\hline Zill & $14,82 \mathrm{a}$ & $22,42 \mathrm{a}$ & $15,00 \mathrm{~b}$ & 24,66 a & 71,40 \\
\hline Irwin & $12,45 \mathrm{a}$ & $18,29 \mathrm{~b}$ & $12,34 \mathrm{a}$ & $18,41 b$ & 75,49 \\
\hline Locale & $12,87 \mathrm{a}$ & $23,94 a$ & $13,46 \mathrm{a}$ & $23,87 \mathrm{a}$ & 81,62 \\
\hline
\end{tabular}

Pour une même colonne, les valeurs suivies d'une même lettre ne sont pas significativement différentes (5\%). 


\begin{tabular}{|c|c|c|c|c|c|c|c|}
\hline Variété de mangue & $\mathrm{pH}$ & $\begin{array}{l}\text { Acidité titrable } \\
\left(\mathrm{mEq} \cdot 100 \mathrm{~g}^{-1}\right)\end{array}$ & $\begin{array}{l}\text { Teneur en eau } \\
(\%)\end{array}$ & $\begin{array}{l}\text { Extraits secs solubles } \\
\text { ('Brix) }\end{array}$ & $\begin{array}{c}\text { Sucres réducteurs } \\
(\%)\end{array}$ & $\begin{array}{c}\text { Vitamine C } \\
(\mathrm{mg} \cdot 100 \mathrm{~g}-1)\end{array}$ & $\begin{array}{c}\text { Fibres } \\
(\%)\end{array}$ \\
\hline \multicolumn{8}{|c|}{ Maturité commerciale } \\
\hline Amélie & & $67,17 \mathrm{a}$ & $16,82 a$ & $85,67 \mathrm{a}$ & $10,83 \mathrm{~b}$ & $266,56 \mathrm{a}$ & $0,70 \mathrm{c}$ \\
\hline Zill & $4,02 \mathrm{~b}$ & 53,92 a & $17,55 \mathrm{a}$ & $80,00 \mathrm{a}$ & 16,32 a & $25,15 d$ & $1,09 \mathrm{~b}$ \\
\hline Irwin & $4,10 \mathrm{~b}$ & $41,75 b$ & $17,15 \mathrm{a}$ & $83,33 \mathrm{a}$ & $17,52 \mathrm{a}$ & $144,15 b$ & $1,07 \mathrm{~b}$ \\
\hline Locale & $4,07 \mathrm{~b}$ & $35,33 \mathrm{~b}$ & $18,26 \mathrm{a}$ & $85,00 \mathrm{a}$ & 18,18 a & $65,55 \mathrm{c}$ & $1,77 \mathrm{a}$ \\
\hline \multicolumn{8}{|l|}{ Maturité avancée } \\
\hline Amélie & $4,55 \mathrm{a}$ & $17,92 \mathrm{c}$ & $17,16 \mathrm{a}$ & 89,17 a & $16,35 \mathrm{~b}$ & $232,38 \mathrm{a}$ & - \\
\hline Zill & $4,19 \mathrm{~b}$ & $34,00 \mathrm{~b}$ & 23,46 a & $80,00 \mathrm{a}$ & $17,93 \mathrm{~b}$ & $13,14 \mathrm{~d}$ & - \\
\hline Irwin & $4,32 \mathrm{a}$ & $27,33 \mathrm{c}$ & $18,92 \mathrm{a}$ & 84,17 a & $24,90 \mathrm{a}$ & $105,50 \mathrm{~b}$ & - \\
\hline Locale & $4,09 \mathrm{~b}$ & $40,92 \mathrm{~b}$ & $20,12 \mathrm{a}$ & $86,67 \mathrm{a}$ & $13,75 \mathrm{c}$ & $67,86 \mathrm{c}$ & - \\
\hline
\end{tabular}

liés à la nature climactérique sont assez marqués chez les fruits tropicaux et particulièrement chez la mangue.

L'analyse de la composition chimique des lamelles de mangue séchée en séchoir électrique n'a pas permis de noter de différences significatives de la teneur en eau résiduelle d'une variété à l'autre (tableau III). Ce taux a varié de $(16,82$ à $18,26) \%$ pour les mangues récoltées à maturité commerciale et de $(17,16$ à $23,46) \%$ pour les fruits ayant atteint une maturité avancée. Selon la littérature, pour le séchage des fruits, une teneur en eau résiduelle de (15 à 28) \% serait recommandée [13]. Les variétés Amélie, Zill et Irwin auraient des teneurs en sucres réducteurs plus élevées dans les lamelles de fruits récoltés à maturité avancée que dans celles des mangues à maturité commerciale. Cette tendance avait également été observée dans le cas des fruits frais. En revanche, les teneurs en vitamine C, significativement différentes d'une variété à l'autre, sont plus faibles dans les fruits à maturité avancée. Cette baisse du taux de vitamine $\mathrm{C}$ pourrait être liée au brunissement non enzymatique poussé des mangues à maturité avancée, ainsi qu'aux réactions de Maillard [14, 15]. Quelle que soit la maturité du fruit, la pulpe des mangues Zill séchées a été la plus pauvre en vitamine $C$ et celle des mangues Amélie, la plus riche.

En séchage solaire, les rendements en matière sèche de la pulpe des fruits a varié de $\left(16,38\right.$ à 24,66) $\mathrm{kg} \mathrm{ms} \cdot 100 \mathrm{~kg}^{-1}$ de $\mathrm{mf}$ (tableau II). Pendant les mois de mai et juin, en intersaison, le produit sèche bien car, en plus des températures élevées $\left(30\right.$ à $\left.43^{\circ} \mathrm{C}\right)$, l'air ambiant est relativement sec puisque l'humidité relative est alors comprise entre (40 et 68) \% ; cela entraîne un fort pouvoir déshydratant. À partir du mois de juillet, avec l'arrivée des pluies, l'air se charge d'humidité, ce qui diminue la vitesse de séchage. Néanmoins, jusqu'en 
fin juillet, le séchage solaire est encore possible, mais la qualité des produits obtenus n'est plus homogène. Le séchage solaire direct affecte l'intensité de la couleur de la mangue séchée. Les risques de pertes dues au brunissement des lamelles (figure 3) augmentent. Selon Pablo [16], une réduction de l'exposition solaire par utilisation de séchoir hybride permettrait de réduire le brunissement des lamelles.

Lorsque le séchage est bien conduit, le produit fini est souple; de couleur jaune foncé ou doré, il ne colle pas aux doigts. Parfois, des lamelles de mangue brun foncé, peu attractives, peuvent apparaître dans les lots secs, mais en faible proportion. Cependant, il arrive parfois que le produit brunisse exagérément et même noircisse. Cela a pu être observé avec des mangues récoltées à maturité avancée lorsqu'elles étaient soumises au séchage solaire, avec une exposition de moins de 5 h d'ensoleillement au début des opérations de séchage. Il semblerait alors que la durée de rayonnement du premier jour du séchage soit importante pour la qualité finale du produit.

L'analyse de la composition chimique des mangues Amélie et Irwin séchées au soleil a montré que le produit obtenu était

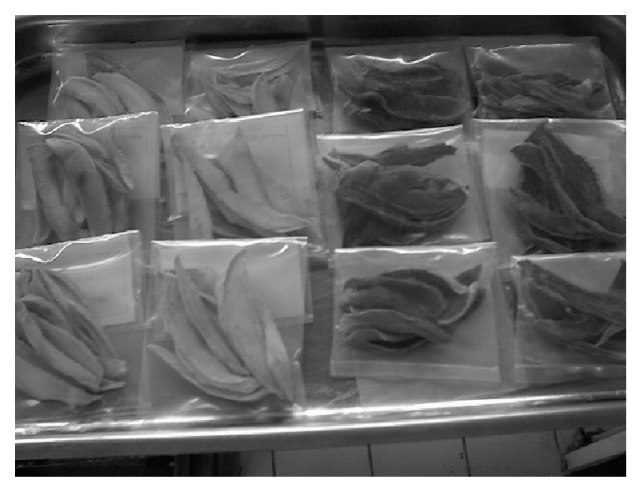

Figure 3.

Coloration des manques séchées : à gauche, mangues séchées à maturité commerciale ; à droite, brunissement des mangues séchées à maturité avancée (Cameroun, photo Irad 2002). similaire à celui obtenu par traitement au séchoir électrique (tableau IV). Cependant, les teneurs en eau restent relativement élevées et donnent des produits finis légèrement plus humides que ceux issus du séchage électrique. Cela expliquerait l'observation de rendements au séchage relativement élevés. Les mangues séchées à maturité physiologique donnent les produits les plus acides et les moins sucrés, mais les plus riches en vitamine $\mathrm{C}$, du moins en ce qui concerne les fruits de la variété Amélie.

Après traitement en séchoir électrique, les taux moyens de conservation des éléments chimiques analysés dans la mangue

\section{Tableau IV.}

Influence du degré de maturité sur la composition chimique de lamelles séchées de mangues Amélie et Irwin après exposition au soleil pendant $27 \mathrm{~h}$ (Cameroun).

\begin{tabular}{|c|c|c|c|c|c|c|}
\hline Variété de mangue & $\mathrm{pH}$ & $\begin{array}{l}\text { Acidité titrable } \\
\left(\mathrm{mEq} \cdot 100 \mathrm{~g}^{-1}\right)\end{array}$ & $\begin{array}{c}\text { Teneur en eau } \\
(\%)\end{array}$ & $\begin{array}{l}\text { Extraits secs solubles } \\
\qquad\left({ }^{\circ} \text { Brix }\right)\end{array}$ & $\begin{array}{c}\text { Sucres réducteurs } \\
(\%)\end{array}$ & $\begin{array}{l}\text { Vitamine C } \\
\left(\mathrm{mg} \cdot 100 \mathrm{~g}^{-1}\right)\end{array}$ \\
\hline \multicolumn{7}{|c|}{ Maturité physiologique } \\
\hline Amélie & $3,63 \pm 0,11$ & $91,50 \pm 18,56$ & $18,20 \pm 5,06$ & $77,50 \pm 5$ & $14,75 \pm 0,49$ & $287,63 \pm 50,97$ \\
\hline Irwin & 3,92 & $51,00 \pm 2,83$ & $15,10 \pm 2,83$ & 60,00 & 16,00 & $116,22 \pm 6,85$ \\
\hline \multicolumn{7}{|c|}{ Maturité commerciale } \\
\hline Amélie & $3,66 \pm 0,11$ & $79,33 \pm 14,28$ & $23,36 \pm 2,20$ & $79,17 \pm 3,76$ & $17,02 \pm 3,39$ & $162,18 \pm 60,65$ \\
\hline Irwin & $4,03 \pm 0,01$ & $46,50 \pm 2,08$ & $15,21 \pm 1,37$ & 80,00 & $19,08 \pm 2,38$ & $137,77 \pm 14,28$ \\
\hline \multicolumn{7}{|l|}{ Maturité avancée } \\
\hline Irwin & $4,06 \pm 0,01$ & $36,25 \pm 0,35$ & $20,14 \pm 0,35$ & 90,00 & $19,65 \pm 1,77$ & $75,66 \pm 1,51$ \\
\hline
\end{tabular}




\section{Tableau V.}

Taux de conservation (\%) de quelques constituants chimiques de la mangue séchée par rapport à leur teneur dans la mangue fraîche après traitement soit par séchoir électrique $\left(24 \mathrm{~h}\right.$ à $\left.50^{\circ} \mathrm{C}\right)$, soit par séchage solaire $(27 \mathrm{~h}$ d'exposition au rayonnement solaire).

\begin{tabular}{|c|c|c|c|c|c|c|}
\hline Type de conservation & Acidité titrable & Teneur en eau & Extraits secs solubles & $\begin{array}{l}\text { Sucres } \\
\text { réducteurs }\end{array}$ & Vitamine C & Fibres \\
\hline \multicolumn{7}{|l|}{ Séchoir électrique } \\
\hline $\begin{array}{l}\text { Conservation } \\
\text { maximale }\end{array}$ & 97,73 & 6,84 & 99,77 & 75,14 & 97,71 & 24,43 \\
\hline $\begin{array}{l}\text { Conservation } \\
\text { moyenne }\end{array}$ & 78,26 & 4,83 & 90,64 & 56,75 & 65,99 & 16,19 \\
\hline $\begin{array}{l}\text { Conservation } \\
\text { minimale }\end{array}$ & 47,73 & 3,09 & 81,29 & 35,39 & 19,89 & 1,47 \\
\hline \multicolumn{7}{|l|}{ Séchage solaire } \\
\hline $\begin{array}{l}\text { Conservation } \\
\text { maximale }\end{array}$ & 98,08 & 4,83 & 96,50 & 76,20 & 71,77 & - \\
\hline $\begin{array}{l}\text { Conservation } \\
\text { moyenne }\end{array}$ & 89,54 & 4,30 & 89,00 & 67,97 & 49,21 & - \\
\hline $\begin{array}{l}\text { Conservation } \\
\text { minimale }\end{array}$ & 84,34 & 3,49 & 78,55 & 54,73 & 23,53 & - \\
\hline
\end{tabular}

séchée ont été supérieurs à 56 \% pour tous les constituants sauf pour le taux en fibres qui a été voisin de $16 \%$ (tableau V). De fortes variations sont apparues selon l'élément considéré. Dans la littérature, des pertes de vitamine $\mathrm{C}$ de l'ordre de $80 \%$ ont été reportées après les opérations de séchage de la mangue [17]. Les enzymes naturellement présentes dans le fruit restent actives pendant le séchage et continuent à synthétiser des produits d'oxydation ou d'hydrolyse. Des facteurs externes tels l'exposition des lamelles par rapport à la circulation de l'air, leur position sur les claies et, probablement, leur épaisseur seraient de nature à stimuler des réactions enzymatiques.

Les lamelles de mangue séchée naturellement par exposition au soleil ont présenté des caractéristiques de conservation des constituants chimiques du fruit frais analogues à celles obtenues après passage en séchoir électrique. Cependant, leur teneur relativement pauvre en vitamine $\mathrm{C}$ pourrait être due à une oxydation de la vitamine suite au rayonnement solaire.

\section{Discussion}

Les résultats obtenus montrent que le séchage peut être utilisé comme une méthode efficace de conservation de la mangue dans le nord du Cameroun. Malgré les différences entre les variétés (forme, poids et couleur), les produits obtenus par séchage sont semblables pour des fruits prélevés à un même degré de maturité. Les rendements de séchage relativement élevés sont acceptables. Les teneurs en eau de la mangue séchée, situées entre (15 et 23) \%, permettent de la classer comme produit à teneur en eau intermédiaire [18] et, de ce fait, stable à température ambiante. Par ailleurs, à cette même température, les pH inférieurs à 4,5 lui assurent une grande stabilité micro-biologique. Du point de vue chimique, les mangues séchées obtenues par séchage solaire direct se sont révélées semblables à celles obtenues après séchage par séchoir électrique [5]. Ces résultats confirment ceux de travaux antérieurs [19] axés sur les possibilités offertes par le séchage pour la conservation des fruits 
tropicaux et particulièrement de la mangue. Toutefois, des problèmes de qualité existent. Le brunissement des lamelles de mangue à maturité avancée pendant le séchage constitue une perte économique pour le producteur. L'utilisation des agents protecteurs tels que du [glycérol + sucre] [18] ou le métabisulfite [20] pourrait améliorer la couleur des mangues séchées. Mais les produits ainsi obtenus perdent la qualité "bio " très recherchée sur les marchés de l'hémisphère nord.

Une durée de séchage en séchoir électrique de $24 \mathrm{~h}$ à $50{ }^{\circ} \mathrm{C}$ [7] correspond à un cycle de traitement d'un lot de tranches séchées. Après ce laps de temps, le séchoir peut être libéré pour accueillir d'autres lots de lamelles de mangue. Une surcharge du four (environ $6 \mathrm{~kg}$ de pulpes $\cdot \mathrm{m}^{-2}$ ou plus) entraîne une plus longue durée de séchage, avec des possibilités de risques d'altération du produit.

Le séchage au soleil donne des produits qui, du point de vue physico-chimique, se rapprochent de ceux obtenus par séchage électrique. Néanmoins, le temps de séchage est plus long. Les produits alors issus du séchage doivent être bien emballés et conservés à l'abri de l'humidité, de la lumière et des insectes.

Finalement, la conservation de la mangue par séchage pourrait donc être réalisée aussi bien à l'échelle industrielle avec utilisation de séchoirs électriques, qu'à l'échelle artisanale par emploi par les petits producteurs des zones rurales démunis d'énergie électrique de la technique de séchage solaire.

\section{Conclusion}

Les variétés de mangue cultivées au nord du Cameroun qui ont été testées au cours des expérimentations présentées se prêtent bien au séchage. Le séchage solaire direct de la mangue est possible et donne des résultats acceptables. Cependant les risques de brunissement sont plus grands qu'en cas d'utilisation de séchoirs électriques, surtout quand les fruits utilisés sont à maturité avancée. Les mangues destinées au séchage doivent donc être récoltées à maturité commerciale pour s'assurer d'une bonne qualité du produit obtenu.

Le séchage de la mangue donne un produit apparemment sain dont les caractéristiques de conservation, d'utilisation et de commercialisation devront être spécifiées.

\section{Remerciements}

Les auteurs remercient le Prasac (Pôle de recherches appliquées aux savanes d'Afrique centrale) qui a financé ces travaux.

\section{Références}

[1] Temple L., Quantification des productions et des échanges de fruits et légumes au Cameroun, Cah. Agric. 10 (2001) 87-94.

[2] Hulme A.C., The mango, in: Hulme A.C (Ed.), The biochemistry of fruits and their products, Vol. 2, Acad. Press, London, UK, 1971, pp. 233-254.

[3] François M., Transformation des fruits tropicaux, GRET, Minist. coop., CTA, ACCT, Collect. Le point sur les technologies, Paris, France, 1993.

[4] Besuchet E., Pury de P., Guide pour la préparation des fruits tropicaux, Féd. Int. Croix Bleue, FICB, Lindenrain, 1998.

[5] Uzuegbu J.O., Ukeka C., Osmotic dehydration as a method of food preserving fruits to minimize ascorbic acid loss, J. Food Agr. 1 (1987) 187-188.

[6] Desmorieux H., Le séchage en zone subsaharienne : une analyse technique à partir des réalités géographiques et humaines, Inst. Natl. Polytech. Lorraine, thèse, Génie des procédés, Nancy, France, 1992.

[7] Candelaria N.M., Raymondo L.C., Comparative drying and reconstitution characteristics of some fruits and vegetables, Philipp. Agriculturist 77 (1994) 321-326.

[8] Sagar V.R., Khurdiya D.S., Effect of ripening stages on the quality of dehydrated ripe mango slices, J. Food Sci. Technol. (India) 33 (1996) 527-529.

[9] Laroussilhe de F., Le manguier, Maisonneuve et Larose, Collect. Tech. agric. prod. trop., Paris, France, 1980. 
[10] Anonyme, Recueil des normes françaises des produits dérivés des fruits et légumes, Jus de fruits, $1^{\text {re }}$ éd., Afnor, Paris, France, 1982.

[11] Tomohiro S., Laboratory manual for food analysis, Techn. coop. proj. Jomo Kenyatta Univ. Coll. Agric. Technol., 1990.

[12] Bergonzini J.C., Duby C., Analyse et planification des expériences, Les dispositifs blocs, Masson, Paris, France, 1995.

[13] O’Mahony J.S., Kahn M.L., Adapa S.N., Fruit infusion using a syrup which has been subjected to enzyme treatment and concentrated, US Patent, US 4626434, 1986.

[14] Cheftel J.C., Cheftel H., Introduction à la biochimie et à la technologie des aliments, $3^{\mathrm{e}}$ éd., Vol. 1., Tech. Doc. Lavoisier, Paris, France, 1980.

[15] Rozis J.F., Sécher les produits alimentaires. Techniques, procédés, équipements, GRET,
CTA, GERES, FAO, Collect. Le point sur, Paris, France, 1995.

[16] Pablo I.S., Solar dryer for tropical fruits and marine products for rural development, NSDB-Technol. J. 4 (1979) 26-41.

[17] Cooke R.D., Breag G.R., Ferber C.E.M., Best P.R., Jones J., Studies of mango processing. I. The foam-mat drying of mango (Alfonso cultivar) purée, J. Food Technol. 11 (1976) 463-473.

[18] Jayaraman K.S., Development of intermediate moisture tropical fruit and vegetable products. Technological problems and prospects. Food preservation by moisture control, Elsevier Appl. Sci. Publ. Ltd., UK, 1988.

[19] Campbell B.A., Campbell C.W., Preservation of tropical fruit by drying, Proc. Fla. State Hortic. Soc. 96 (1983) 229-231.

[20] Sagar V.R., Khurdiya D.S., Studies on the dehydration of Dashehari mango slices, Indian Food Packer 53 (1999) 5-9.

\section{Aptitud al secado de frutos de algunas variedades de mangos cultivados en Camerún.}

Resumen - Introducción. En Camerún, la producción del mango (Mangifera indica) es alta durante el período de cosecha que dura (2 a 3) meses $a_{n ̃ n o}^{-1}$ según las regiones. Esto ocasiona un problema de exceso de producción. Algunos estudios pusieron de manifiesto que el secado del mango sería una vía interesante para la conservación de esta superproducción. En zona rural, los secaderos eléctricos, que permiten un secado rápido y constante, no pueden utilizarse debido a la falta de electricidad. Para incitar a los productores a utilizar el secado como técnica de conservación de los mangos excedentarios en algunas temporadas, se estudió la aptitud al secado de algunas variedades de mangos producidos en Camerún comparando los productos obtenidos mediante secado eléctrico o solar. Material y métodos. Los frutos de tres variedades de mango mejoradas (Amélie, Zill e Irwin) y de una variedad local (Horé Wandou) se recolectaron en dos grados de madurez: madurez comercial y madurez avanzada. Después de preparar la fruta, se cortó la pulpa en laminas y se secó mediante secador eléctrico o por exposición directa al sol. Antes y después de secado, se estudió la composición fisicoquímica de las muestras de pulpa. Resultados y discusión. Tras secado a $50^{\circ} \mathrm{C}$ durante $24 \mathrm{~h}$ (secador eléctrico), las láminas de mango secado tenían un contenido de agua situado entre (16 y 24) \%. Aparecieron diferencias significativas en los rendimientos al secado de las variedades estudiadas. Los contenidos de los distintos constituyentes fisicoquímicos analizados variaron en función de las variedades y el grado de madurez de los frutos cosechados. Las tasas promedio de conservación de los constituyentes (vitamina $\mathrm{C}$, azúcares reductores, extractos secos solubles) tras secado fueron superiores al $56 \%$, exceptuando el contenido de fibras con un porcentaje de conservación del $16 \%$. Los mangos secados directamente al sol fueron similares a los de secadero eléctrico a pesar de una duración de secado mayor y un menor contenido de vitamina C. Conclusión. Las variedades Amélie, Zill, Irwin y la variedad local Horé Wandou muestran buenas aptitudes para el secado, que conserva los principales elementos nutritivos con rendimientos importantes.

Camerún / Mangifera indica (frutas) / variedades / aptitud para la conservación / secado / secadoras / propiedades fisicoquímicas 